\title{
Track Behavior and Crash Risk Analysis of Passenger Cars on Hairpin Curves of Two-Lane Mountain Roads
}

\author{
Zhigang Yu, ${ }^{1}$ Ying Chen, ${ }^{2}$ Xiaobo Zhang, ${ }^{3}$ and Jin $\mathrm{Xu} \mathbb{1 D}^{4}$ \\ ${ }^{1}$ Chengdu Vocational \& Technical College of Industry, No. 818, Da'an Road, Zhengxing Street, Tianfu New District, \\ Chengdu 610218, Sichuan, China \\ ${ }^{2}$ College of Traffic and Transportation, Chongqing Jiaotong University, No. 66, Xuefu Ave., Nan'an District, \\ Chongqing 400074, China \\ ${ }^{3}$ China Railway Siyuan Survey and Design Group Co., Ltd., No. 745, Heping Avenue, Wuchang District, Wuhan 430063, \\ Hubei Province, China \\ ${ }^{4}$ Chongqing Key Laboratory of "Human-Vehicle-Road" Cooperation \& Safety for Mountain Complex Environment, \\ Chongqing Jiaotong University, No. 66, Xuefu Ave., Chongqing 400074, China
}

Correspondence should be addressed to Jin Xu; yhnl272727@163.com

Received 17 April 2021; Revised 28 June 2021; Accepted 30 June 2021; Published 13 July 2021

Academic Editor: Maria Castro

Copyright (C) 2021 Zhigang Yu et al. This is an open access article distributed under the Creative Commons Attribution License, which permits unrestricted use, distribution, and reproduction in any medium, provided the original work is properly cited.

Hairpin curves are often employed in alignment layout and an important feature that identifies dangerous driving conditions for mountain roads. However, driving behaviors at hairpin curves remain ambiguous. Field driving tests were conducted in this study on one two-lane mountain road with 11 hairpin curves. Vehicle-mounted equipment was utilized to collect track and lateral distance between the wheels and the lane markings under naturally driving conditions. Track morphology and patterns, risks, and road crash mechanisms were analyzed. The main findings are as follows. Curve cutting was a typical method for negotiating hairpin curves, was observed for left and right turns, and can be classified into three types based on the location of the cutting point, namely, cutting at curve entry, cutting at curve middle, and cutting at curve exit. Based on the lateral positional relationships between tracks and lane markings, six track patterns are determined for left turns and four track patterns for right turns. When passing a right turn by cutting the curve, a driver occupied the right shoulder of the turn; therefore, there is a risk of colliding with the mountain or the guardrail. When making a left turn into hairpin curves, a driver occupied the right shoulder on curve exit, resulting in running off the road or colliding with the guardrail. More than $70 \%$ and $60 \%$ of drivers occupied the opposite lane when turning right and turning left, respectively, into a hairpin turn, which led to intertwining between the tracks in the two driving directions and therefore a risk of potential collisions.

\section{Introduction}

Mountains (areas with rugged topography, excluding high plains) account for more than one-third of China's geographic area. To adapt to the terrain environment, mountain roads are characterized by a complex alignment combination, a relatively high proportion of bends, and relatively low geometry element values. In mountainous countries, Switzerland, Austria, Japan, South Korea, and China, hairpin curves have always been an alignment combination that is commonly employed when designing mountain roads, particularly two-lane roads. As a result, hairpin curves have become an important, distinctive feature of relatively lowstandard mountain roads. The "24-zag" road in Qinglong, Guizhou, China, is the throat of Stilwell Road and was the transport artery in the China-Burma-India Theater during World War Two, has 24 hairpin curves, as shown in Figure 1(a). Figures 1(b)-1(f) show an additional five wellknown winding mountain roads in China, all of which have dense hairpin curves.

Due to the low traffic volume, drivers have a high degree of freedom when driving on two-lane mountain roads. Many drivers utilize the full width of the road, and their tracks, to some extent, deviate from the centerline of the road (an 


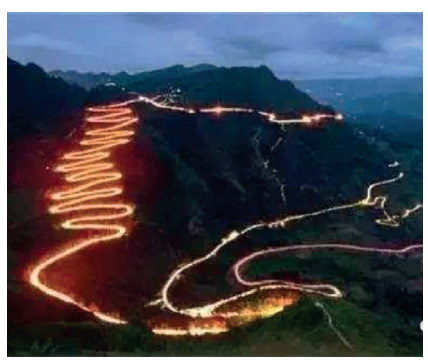

(a)

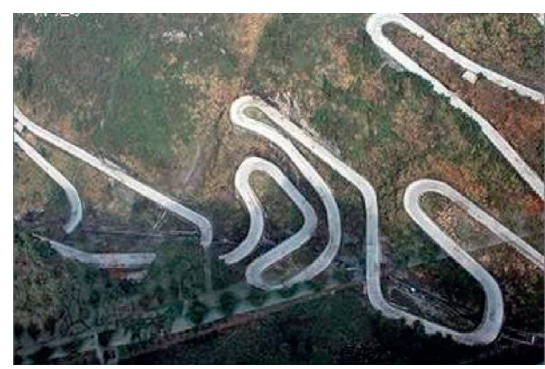

(d)

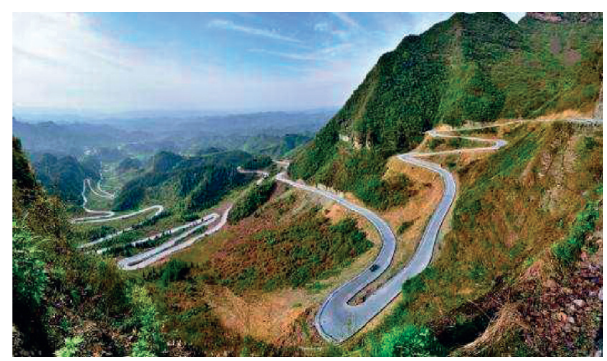

(b)

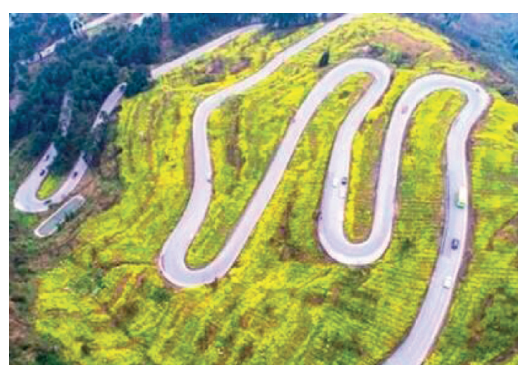

(e)

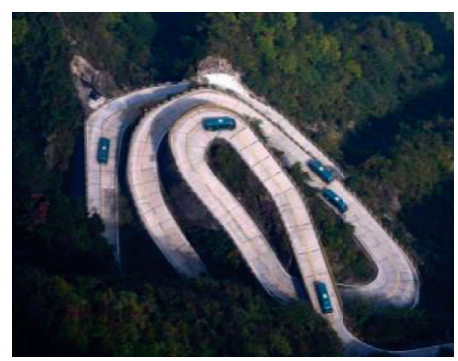

(c)

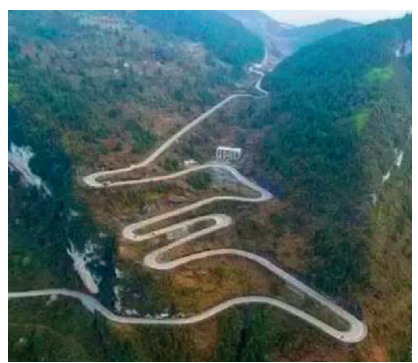

(f)

Figure 1: Winding mountain roads with dense turns in China.

imaginary driving track in road design) [1]. In recent years, accidents that involve vehicles that deviate from traffic lanes in the curved sections of mountain roads (e.g., collisions and scratching between vehicles in opposite directions, overturns, collisions with the guardrail or mountain, and running off the road) have occurred frequently and even increased. These collisions comprise the primary types of traffic accidents that occur on mountain roads. Hairpin curves are an important component of two-lane mountain roads. However, driving patterns at hairpin curves remain ambiguous. Thus, a thorough study of vehicle tracks in hairpin-turn sections, analysis of the adaptation and interference between tracks and lanes markings, calculation of road crash risks, and interpretation of road crash mechanism are necessary to provide a scientific basis and theoretical support for traffic safety facility design, manage traffic operations, and improve traffic safety in dangerous road sections.

1.1. Literature Review. In recent years, measurements of driving behaviors in hairpin-turn sections were rarely reported. Discetti [2] used a camera and traffic counter to record the driving process of the vehicle along hairpin curves, and spot speed on the equal interval cross sections and the average acceleration/deceleration between adjacent sections are obtained.

But for general road curves, in the past decades, many studies have been done on the vehicle track behavior. Based on the research methods, three types of research on vehicle tracks and driving behaviors on roads are underway.

The first aspect is analysis of the lateral positions of tracks based on video images. Chapuis et al. [3] extracted the track in real time by extracting the lane markings on both sides of the vehicle from images of the front of the vehicle captured by a vehicle-mounted camera and estimating the lateral position of the vehicle, and track patterns were then classified. Llorca et al. [4]; Kanagaraj et al. [5]; and Raju et al. [6] extracted vehicle tracks from captured images and examined driving behaviors (e.g., overtaking and lane changing, lateral positions, headway, and relative speed) or vehicle operation parameters and described the operating status of traffic. Xu et al. [1] and Deng et al. [7] conducted field driving tests on two-lane mountain roads in naturally driving conditions and determined vehicle tracks based on Global Positioning System (GPS) data combined with video images. In addition, by clustering tracks based on the shape characteristics of lateral deviation ratio curves, they identified the track patterns on the curved sections of mountain roads and analyzed the vehicle crash risks based on the topological relation between tracks and traffic lanes.

The second aspect is investigating the effects of road parameters on track behavior using driving simulators or pure computer simulations. For example, Peng et al. [8] and $\mathrm{Xu}$ et al. [9] simulated the driving processes in the curvefollowing and curve-cutting patterns using a "driver-vehicleroad" simulation system and examined the effects of bend geometric parameters on vehicle track and speed. Shu et al. [10] acquired the steering distance and duration of a minibus when entering and departing from single-curve bends, and the relationships of these two parameters with curve radius and deflection angle and vehicle wheelbase were analyzed.

The third aspect is collecting vehicle track data on a preselected cross section by roadside observations. $\mathrm{Nu}$ merous methods are available for track data collection. For example, scale marks can be painted on pavement, and then a camera can be used to film moving vehicles. Thus, the lateral distances between the vehicles and the road boundaries can be determined [2]. Alternatively, vehicles can be detected using infrared methods on the roadside, and 
subsequently, their lateral positions relative to the road boundaries can be determined [11]. Fitzsimmons et al. [12] collected track data at five stations within the horizontal curves in both directions of travel using pneumatic tubes fixed on pavement and analyzed the characteristic of vehicle trajectories and speed along horizontal curves. This method can be employed to obtain track and position data for a relatively large number of samples; however, it can only be applied to determine the relative lateral positions of vehicles on preselected sections (entrance, middle, and exit of a turn) and cannot be applied to continuously follow the track of a single vehicle.

Based on the lateral position or lateral deviation of the vehicles, as well as their secondary indicators, vehicle tracks were classified into different types in some works of literature. For example, Spacek $[11,13]$ identified the trajectories within curved areas into six types according to the lateral positions of vehicles in seven cross sections (mainly the position relationship between trajectories and lane markings), that is, ideal, normal, correcting, cutting, swinging, and drifting, in which "cutting" is the major behavior on curves, and the safety of each track type was analyzed, as well as the influence of driving speed and curve geometry on the trajectory shape. Following Spacek's work, Geedipally and Pratt [14] developed a series of models to predict different trajectory types within horizontal curves as a function of curve geometric, pavement, and traffic control characteristics. Mauriello et al. [15] used a driving simulator to obtain the driving process of 50 drivers on curved sections with different radii, and 2000 trajectories were recorded. Based on the lateral position of the vehicle at the entrance, middle, and exit of the curve, similarly, six trajectory types were identified, among which four track types are identical with classification results of Spacek's work; moreover, their results also show that the increase of curve radius will reduce the probability of unsafe driving behavior. In a literature review by Boruah and Maurya [16], vehicle lateral placement and track behavior on horizontal curves were summarized, indicating that the lateral position of vehicle within a curve can affect the side frictional requirement and thus further affect the lateral stability and safety of the vehicle.

While these studies employ various methods to analyze single- and multivehicle tracks on roads, they focus on the identification of lane-changing behaviors in tangents, lateral deviations in normal horizontal curved sections, and track pattern identification. Relatively field driving tests in natural driving conditions in hairpin-turn sections of mountain roads have not been reported. As a result, actual track morphology and patterns at hairpin curves are not comprehensively understood. In addition, limited by the positioning accuracy of GPS devices, previous studies were unable to analyze the morphology of tracks on mountain roads based on single-source GPS data. Moreover, existing research related to vehicle trajectory rarely involved hairpin curves, so far the track shape and driving safety along hairpin curves have not been known.

In this study, field driving tests were performed in natural driving conditions. Continuous vehicle tracks were collected on mountain highway using high-precision GPS devices. Subsequently, the tracks in the hairpin-turn sections were extracted. This approach avoids relatively large original errors in estimating lateral positional deviations. Based on the topological relation between tracks and road boundaries, vehicle tracks were clustered, and the track patterns were classified. Afterwards, based on the interference between tracks and road boundaries, crash risks, accident-prone areas, and crash types were determined. Thus, hairpin-turn sections were accurately analyzed in terms of operating conditions, driving behaviors, and crash mechanisms.

\section{Methods}

To obtain accurate vehicle track and visually reflect the track-selection behaviors of drivers in hairpin-turn sections of mountain roads, field driving tests were performed on a mountain highway with complex alignment. Centimeterscale-precision vehicle-mounted GPS devices were employed to collect vehicle track data in natural driving conditions. By cluster analysis, typical track patterns were identified, and the road crash risk for each track pattern was analyzed.

2.1. Test Road and Subjects. The section of the National Highway G211 from Huadiwan to Ningjiazhai in Pengshui County, Chongqing, China, was selected for the driving tests, as shown in Figure 2(a). The design speed for this road (Class IV mountain road) is $20 \mathrm{~km} / \mathrm{h}$. This road section is in satisfactory condition with clear signs and markings. Lane width in tangent is $3.0 \mathrm{~m}$; width in hairpin curve areas is $1.25 \mathrm{~m}$; superelevation rate is $5 \%$; and hardened shoulder on both sides is $0.75 \mathrm{~m}$ (including $0.15 \mathrm{~m}$ lane marking), as shown in Figures 2(b) and 2(c). The test section has a total length of $9.7 \mathrm{~km}$ and 11 hairpin curves. Table 1 summarizes the geometric parameters. The hairpin curves usually adopt the minimum radius allowed by the relevant design standards, and from the table, we can notice the radius of the 11 hairpin curves around 20 meters. For each hairpin curve, a spiral is used to connect the tangent and the circular curve at both sides of the circular, and the length of the spiral used in each hairpin curve is $25 \mathrm{~m}$. The traffic flow on this road is relatively low, and civilian residences are scattered along both sides of the road; however, few roadside pedestrians utilize the road; therefore, roadside interference is rare, and drivers are able to drive freely along the road.

The tests were conducted in November 2019. A total of 20 drivers (15 males and five females) participated in the tests. These drivers had 4-30 years of driving experience with an average of 11.93 years of driving experience. Cumulatively, these drivers had driven $1.8-20 \times 10^{4} \mathrm{~km}$ and had driven an average of $8.4 \times 10^{4} \mathrm{~km}$. The ages of these drivers ranged from 22 to 48 years, with an average age of 29.95 years. The drivers were recruited from the community; each driver drove a round trip in the test road once or twice and received a compensation of CNY 200. Before the driving test, each driver was asked to complete a driving behavior questionnaire to determine his or her basic information and driving style. 


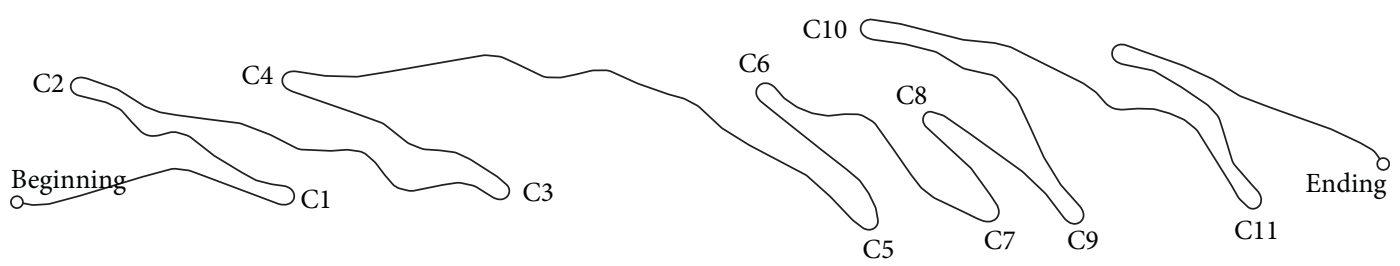

(a)
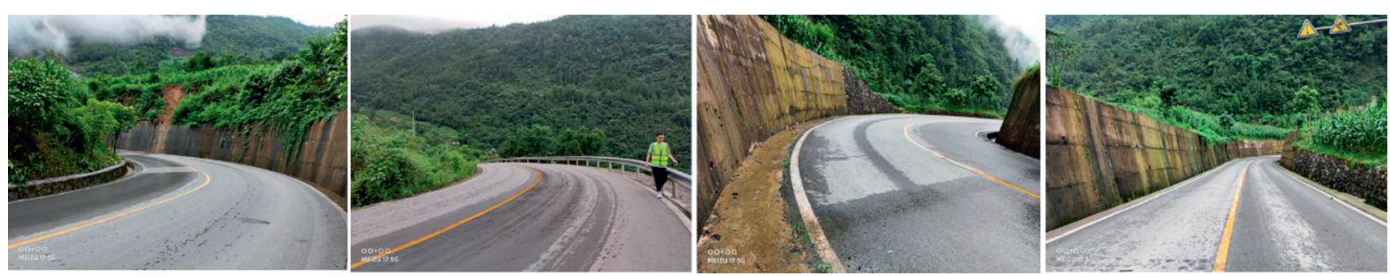

(b)
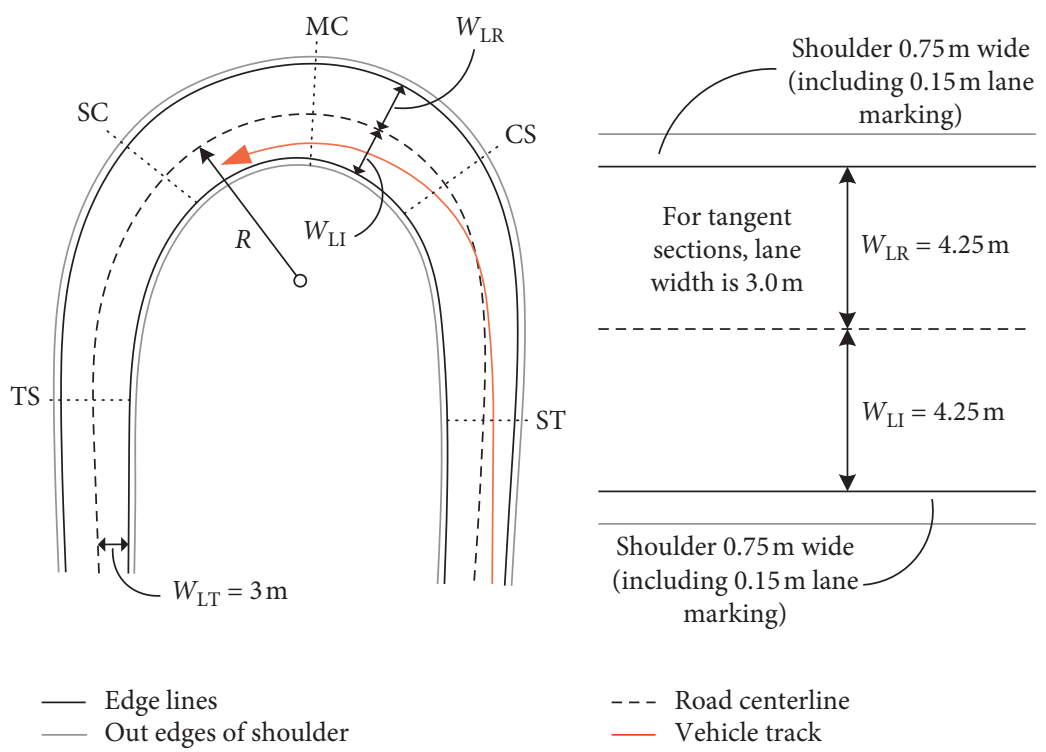

TS, point of change from tangent to spiral

SC, point of change from spiral to circular

$\mathrm{MC}$, middle of the circular CS, point of change from circular to spiral

ST, point of change from spiral to tangent

$W_{L T}$, lane width on tangent

$W_{L O}$, outside lane width on circular curve

$W_{L I}$, inside lane width on circular curve

(c)

Figure 2: The test road. (a) Test road section. (b) Pavement, lane marking, and roadside environment. (c) Geometry parameters of hairpin curves.

TABLE 1: Geometric parameters of the hairpin curves.

\begin{tabular}{|c|c|c|c|c|c|}
\hline No. & Lane width $(\mathrm{m})$ & Curve radius $(\mathrm{m})$ & Deflection angle $\left({ }^{\circ}\right)$ & Curve length (m) & Gradient (\%) \\
\hline 1 & $4.25,4.25$ & 20.156 & 192.73 & 92.975 & 3 \\
\hline 2 & $4.25,4.25$ & 20.233 & 186.19 & 90.797 & 3 \\
\hline 3 & $4.25,4.25$ & 20.079 & 180.09 & 88.138 & 3 \\
\hline 4 & $4.25,4.25$ & 20.029 & 169.51 & 84.381 & 3 \\
\hline 5 & $4.25,4.25$ & 20.425 & 159.43 & 87.519 & 2.7 \\
\hline 6 & $4.25,4.25$ & 20.861 & 190.62 & 94.561 & 2.8 \\
\hline 7 & $4.75,3.55$ & 22.315 & 150.6 & $83.815 /$ & 3 \\
\hline 8 & $4.25,4.25$ & 20.26 & 172.64 & 86.202 & 3 \\
\hline 9 & $4.25,4.25$ & 20.304 & 186.58 & 91.257 & 3 \\
\hline 10 & $4.25,4.25$ & 20.327 & 180.54 & 89.188 & 3 \\
\hline 11 & $4.25,4.25$ & 20.347 & 194.54 & 94.259 & 3 \\
\hline
\end{tabular}

2.2. Test Equipment and Vehicles. Mobileye ME630 system was employed to collect data at a sampling frequency of $10 \mathrm{~Hz}$ for the lateral distance between the wheels on both sides of a vehicle and the traffic lanes when the vehicle was moving. SV-MDOO9HD, a vehicle-mounted camera (tachograph), was utilized to record video and audio data throughout the trip. SPEEDBOX, an on-board inertial measurement system, was used to acquire high-accuracy 
vehicle information (including location, attitude, speed, and acceleration) in real time with a sampling frequency of $100 \mathrm{~Hz}$. A Buick GL8 was applied as the test vehicle. In addition, a computer was employed to store the data acquired by the Mobileye system and SPEEDBOX. The above instruments used in driving tests are shown in Figure 3.

2.3. Test Scheme and Procedure. Before starting the driving test, each driver was asked to operate the vehicle in their favorite way, that is, to maintain his or her natural driving state. Each driver drove a round trip in the test section once or twice. In addition, each driver was accompanied in the vehicle by two testers, who were tasked with recording the starting time of the driving test and the time at which the vehicle-mounted equipment began to record data and, to the maximal extent, ensuring that the two time points coincided with one another. Each driver drove in the road section by following the predetermined test route and subsequently returned to the starting point. Thus, he or she completed a closed driving loop. Then, the driver stopped the vehicle, and the tester stopped recording, stored various data, and recorded the time when the vehicle stopped.

During the data postprocessing process, the tracks at each U-turn were separately analyzed. The tracks were also compared with the videos captured by the vehicle-mounted recorder. Data when the vehicle is seriously disturbed by roadside interference or obstacles above the road were removed (e.g., the vehicle in front moved slowly and the test driver was unable to overtake it for a long period of time). Moreover, the data do not include lane position when traffic was present in the opposing travel lane, that is, all data based on the subject vehicle without the presence of vehicles in the opposing travel lane.

The track data were obtained using the following procedure: longitude and latitude data output from SPEEDBOX GPS system were transformed to geodetic coordinates; subsequently, each coordinate point was connected with its two adjacent points; thus, a continuous track of the vehicle was obtained. Afterwards, tracks measured from various drivers and road boundaries were superimposed in the same coordinate system.

\section{Morphological Characteristics and Patterns of the Tracks on Hairpin Curves}

3.1. Overall Characteristics of the Tracks within Hairpin Curve Areas. To facilitate the analysis of the track pattern, the tracks of all drivers at the hairpin curves were separately superimposed. Thus, the tracks were segmented, and the track of each driver at each hairpin turn was extracted. After segmentation, the tracks of all the drivers were superimposed within road boundaries on the two sides to facilitate visual observation of the distribution along the hairpin curves. Figures 4 and 5 show the tracks at ten hairpin curves. In the two figures, approximately 20 tracks exist at each hairpin turn, forming a track beam. The distribution width of the track beam can be applied to represent the discreteness of the tracks. The following findings are obtained from
Figures 4 and 5 based on the diameter of track beam, lateral positional relationships between track and the road boundaries, and tightness of track beam:

(1) The width of each track beam varies among the locations at each hairpin turn. For example, the track beam is relatively tight in the middle of the turn and relatively loose at the ends of the turn, as observed in Figures $4(\mathrm{~g}), 5(\mathrm{~d})$, and $5(\mathrm{~g})$. This finding suggests a change in the track-selecting behaviors of the drivers within the curved areas.

(2) The track beam becomes closer to the inner side of each turn after entering its curved segment; this indicates that drivers generally tend to pass the hairpin turn with relatively low traffic flow in a curve-cutting pattern and occupy some of the opposite lanes. The curve-cutting point is marked in Figures 4(b), 4(c), and 4(f). After exiting the turn, most tracks recover to their original lateral positions. However, at some individual turns (e.g., turns 3 and 10 ), some drivers continued to occupy the opposite lane.

(3) Vehicle tracks at right turn reveals that some drivers drove close to the inner side of the turn, as demonstrated in Figure 5; drivers drove close to the inner side of turns 2 and 10. However, a large proportion of the drivers drove close to the centerline of the turn (e.g., turns 1, 5, 6, and 11) and occupied some of the opposite lanes. The risk of collisions with vehicles in the opposite lane remains for a right-turning vehicle that occupies the opposite lane at a hairpin turn. Moreover, some drivers also passed the turn by curve cutting; that is, they first adjusted the track to the outer side of the turn before the curve entrance and then changed the track close to the inner side of the turn (e.g., Figures 5(c), 5(f), and 5(g), where C1 and $\mathrm{C} 2$ are curve-cutting points). Based on the location of the curve-cutting point, the curve-cutting behaviors can be classified into three types, namely, curve cutting at curve entrance, curve cutting at curve middle, and curve cutting at curve exit.

3.2. Classification of Track Patterns. The tracks can be classified based on their lateral positional relationships with the road boundaries and the centerline of the road, and track behavior patterns thus are determined. The criteria for the classification of track types are as follows:

(i) Position of the curve-cutting point: when the vehicle passes through a horizontal curve, some drivers will steer the vehicle to the inside of the curve to obtain a larger radius or a higher speed, namely, cutting the curve. In this paper, the closest point between the path and the inside edge of the curve is defined as the cutting point. For a given hairpin curve, the position of the cutting point may appear at the entrance, middle, or exit of the curve, as shown in Figures 6(a) and 6(b). 

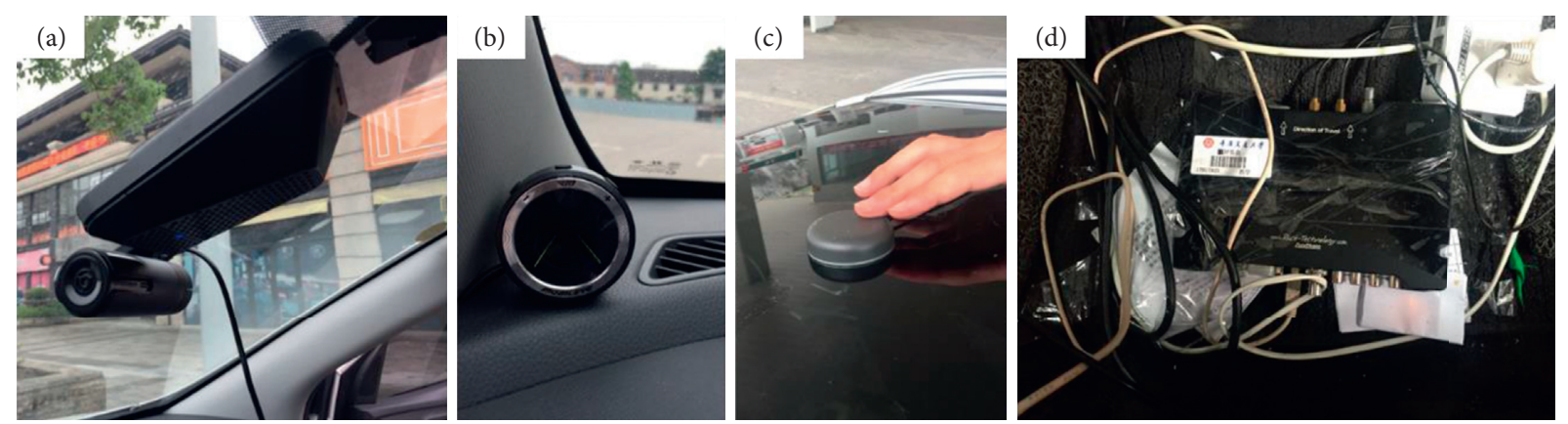

FIGURE 3: On-board apparatus used in driving tests. (a) Mobileye 630; (b) tachograph; (c) antenna of SPEEDBOX; (d) controller of SPEEDBOX.

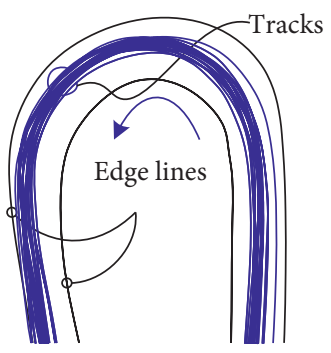

(a)

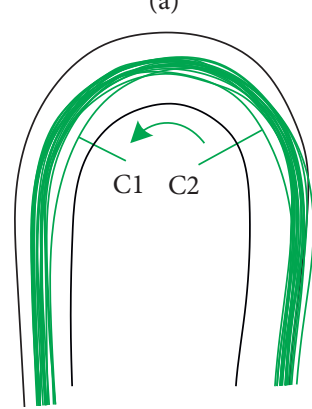

(f)

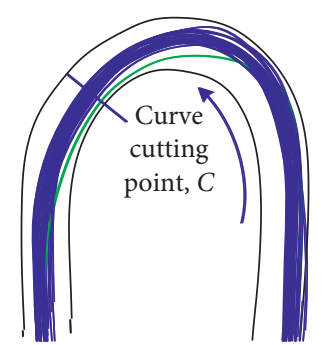

(b)

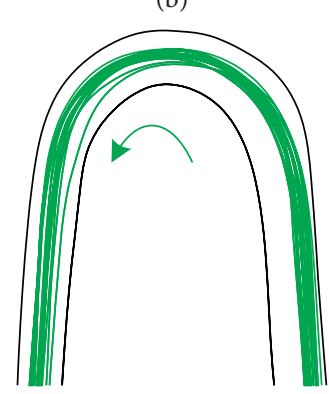

(g)

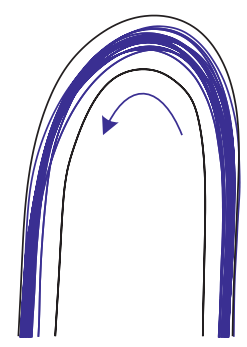

(c)

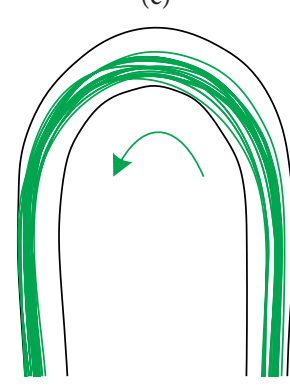

(h)

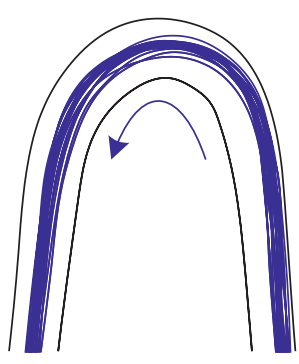

(d)

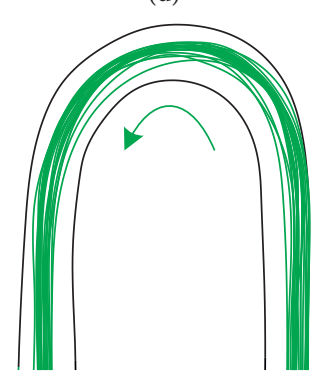

(i)

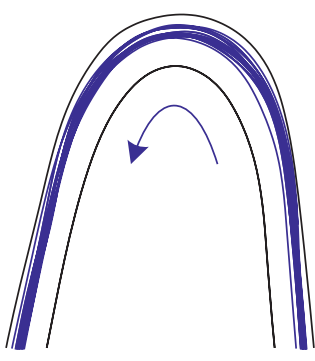

(e)

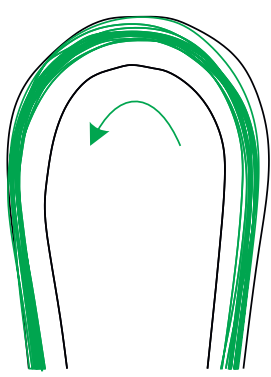

(j)

Figure 4: Left-turning traveling tracks at the hairpin curves. (a) Turn 1. (b) Turn 2. (c) Turn 3. (d) Turn 4. (e) Turn 5. (f) Turn 6. (g) Turn 8. (h) Turn 9. (i) Turn 10. (j) Turn 11.

(ii) Type of lane departure: in Figure 5, within a hairpin curve, the vehicle often leaves the carriageway. For two-lane highways, when the vehicle deviates from its carriageway to the left, it will occupy the opposite lane; on the contrary, when vehicles deviate to the right of the carriageway, they will occupy the hard shoulder on the right. Therefore, we divide lane departure into two types: encroaching on the opposite lane and encroaching on shoulder, as illustrated in Figure 6(b). And for those drivers who follow the rules, they will keep their vehicles within the lane all the time, namely, lane keeping.

(iii) Location of vehicle departing from traffic lane: based on the geometric features of a hairpin turn and track shape, a turn is divided into five areas, namely, tangent prior to the curve (A1), curve entrance (A2), curve middle (A3), curve exit (A4), and tangent after the curve (A5), as shown in Figure 6(c).

Based on the deviation of the track from the lane in various areas, as well as the distribution of the curvecutting point along the curve, vehicle track patterns at hairpin turns can be identified. Here, turn 9 is selected as an example. Left-turning vehicle tracks at this turn can be classified into three patterns, and a notable morphological difference between track shapes with different patterns is observed; the track pattern as illustrated in Figure 7. In the figure, vehicle with pattern $\mathbf{P}_{1}$ travels along road centerline within the first three parts of the curve (A1 A3); as a result, the vehicle body encroaches on the opposite lane in this area and then changes into lane keeping at the last two parts; the vehicle with pattern $\mathbf{P}_{2}$ drove in the lane in the first two parts and occupied the opposite lane in the 


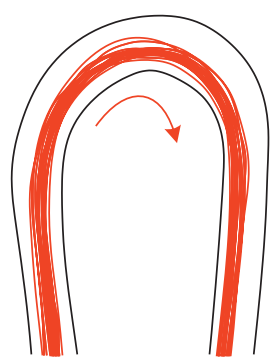

(a)

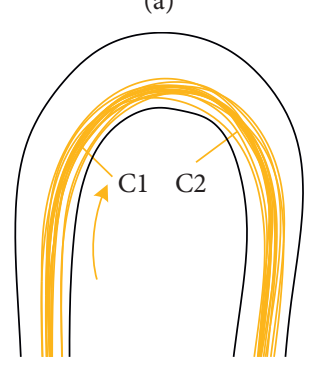

(f)

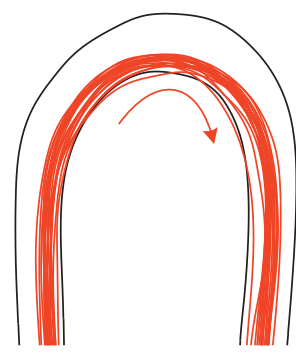

(b)

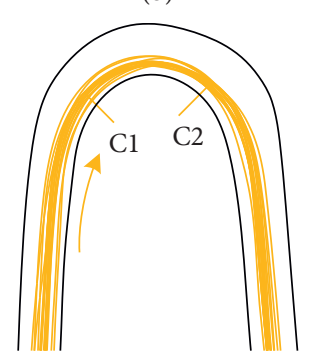

(g)

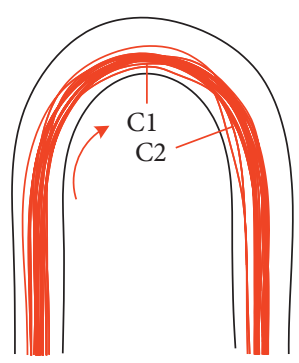

(c)

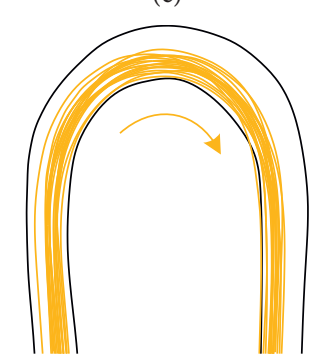

(h)

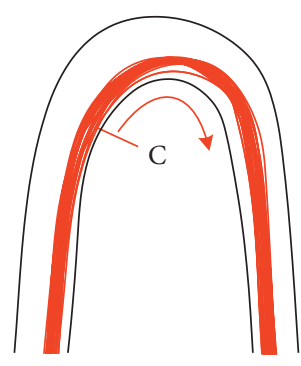

(d)

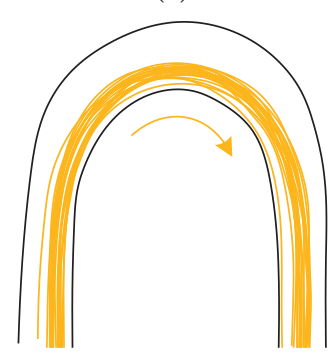

(i)

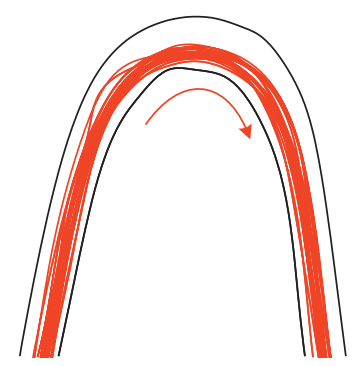

(e)

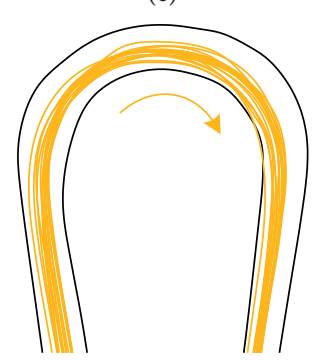

(j)

FIgURE 5: Right-turning traveling tracks at the hairpin curves. (a) Turn 1. (b) Turn 2. (c) Turn 3. (d) Turn 4. (e) Turn 5. (f) Turn 6. (g) Turn 8. (h) Turn 9. (i) Turn 10. (j) Turn 11.

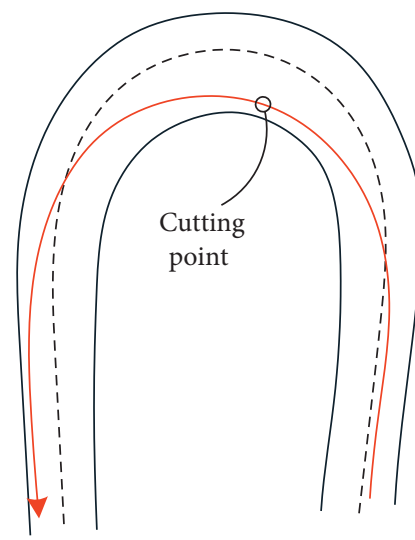

(a)

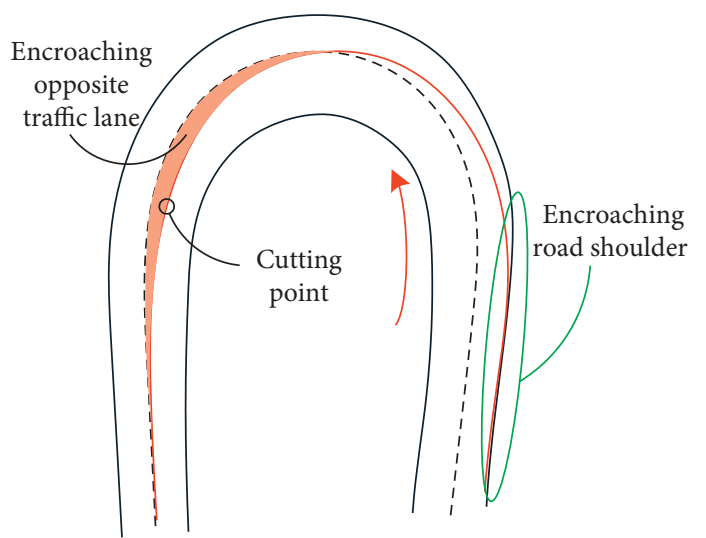

(b)

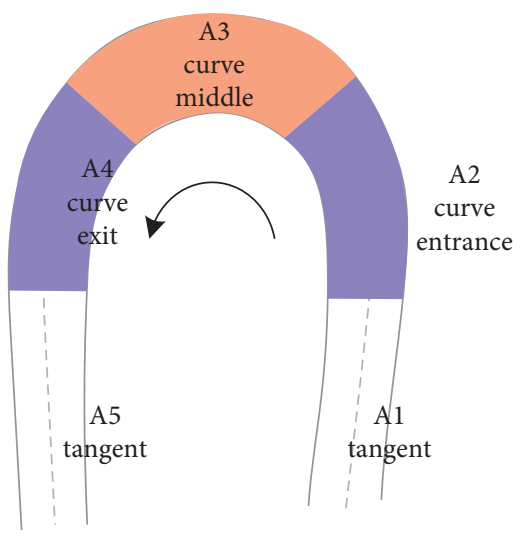

(c)

FIgURE 6: Curve cutting and opposite lane-occupying. (a) The driver cuts a curve near the middle of the curve; (b) vehicle strays from the lane and cuts the curve at curve exit; (c) segmentation of a hairpin curve.

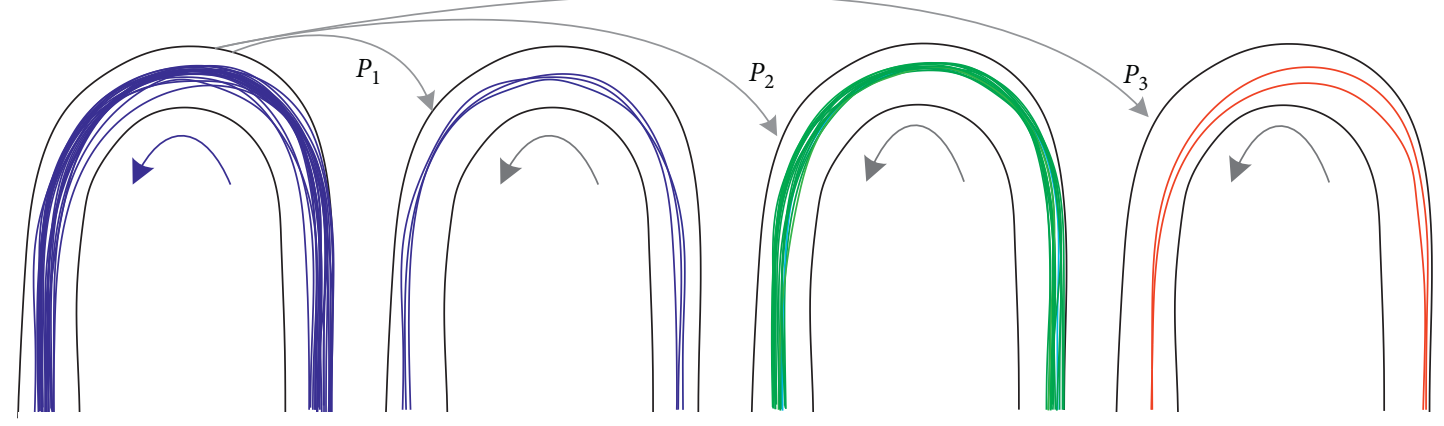

Figure 7: Track morphology and pattern identification at turn 9. 
last three parts; the difference between patterns $\mathbf{P}_{2}$ and $\mathbf{P}_{3}$ is that the vehicle with pattern $\mathbf{P}_{3}$ cuts the curve at curve exit.

3.3. Track Patterns at Left-Turning Curves. When a driver steers his/her vehicle into a left-hand curve, the "standard" driving behavior is to control the vehicle traveling on the outer lane of the turn. The fatal crashes on two-lane mountain roads are that the vehicle deviates from the lane and exposes to the opposite lane or hard shoulder, which can cause serious accidents, such as collisions with another vehicle and running off the road (or collisions with the roadside guardrail). Based on the distribution of locations where the track deviates from the lane, the track behaviors are classified into six patterns, namely, pattern $I_{L} \sim \mathrm{VI}_{L}$, as shown in Figure 8.

In Figure 8, except for the "standard" track pattern (pattern $\mathrm{I}_{L}$, traveling in the legal lane throughout the course) in Figure 8(a), occupying the opposite lane or shoulder at the entrance, the middle, and the exit of the turn can be observed in all the track patterns. In track pattern $\mathrm{II}_{L}$, the drivers drove close to the outer side at curve entrance to obtain an adequate visual range and left the turn by cutting the curve when determining the driving conditions. However, driver often drove into the opposite lane after cutting the curve, and this maneuver could cause a serious road crash risk. This track pattern is observed in professional racing drivers on F1 racing tracks. With pattern $\mathrm{III}_{L}$ in Figure 8(c), the tracks coincided with the centerline of the road before entering the turn; that is, the drivers drove on the centerline. Due to the inertia of the vehicle, the tracks deviated to the outer side, and the vehicle occupied the right-side hard shoulder. The classical curve-cutting behavior in Figure $8(\mathrm{~d})$ is a common track pattern in curved sections of mountain roads, and it is also frequently observed on F1 racing tracks. In Figure 8(e), the curve-cutting point with pattern $V_{L}$ appears before the curve middle and the vehicle occupies the shoulder on the right side when leaving the turn after cutting the curve. The pattern driving on the centerline in Figure $8(\mathrm{f})$ is a common driving pattern observed on mountain roads. We often think that this pattern primarily occurs on tangents; however, this study demonstrates that it also occurs in sharp curves, such as hairpin-turn road sections.

3.4. Track Patterns at Right-Turning Curves. When a driver negotiates a right turn, the "standard" operating behavior is to control the vehicle to travel along the inner side lane. Right turns are generally considered very safe because a driver typically occupies the shoulder on the right side, unlike a left turn, where a driver occupies the opposite lane to cut a curve. Thus, heading-on collisions are avoided. However, some research indicates that heading-on crashes also occur at right turns $[17,18]$; that is, right-turning vehicles also occupy the opposite lane. Based on the lateral positional relationships between the track and the road boundaries, four track patterns at right turns are determined, as shown in Figure 9.

With the pattern $I_{R}$ in Figure 9(a), drivers control the vehicle in the legal lane in the middle of the hairpin turn.
However, before the entry into the turn and after the exit from the turn, track was close to the centerline of the road, which led to some extent of curve-cutting behavior. Driving close to the inner side at a right turn will cause an inadequate sight distance. To acquire a more adequate visual range and ensure a gentler track radius, some drivers will engage in the track pattern $\mathrm{II}_{R}$ shown in Figures 9(b) and 9(c). Specifically, a driver will drive close to the outer side of a turn upon entry by occupying the opposite lane and leave the turn by cutting the curve, which allows a driver to leave a turn at a relatively high speed. In pattern $\mathrm{III}_{R}$ in Figures 9(d) and 9(e), drivers can obtain larger track radii when entering a curve, and the vehicle track deviates from the legal lane due to the vehicle motion inertia. In addition, the vehicle occupies the opposite traffic lane within a relatively long distance. Compared with the track deviation at curve entrance in pattern $\mathrm{II}_{R}$, the risk of track deviation at curve exit is higher because when entering a bend, the vehicle decelerates and better sight conditions exist. In comparison, the vehicle accelerates when departing from a turn, and avoiding vehicles in the opposite direction is more difficult. In pattern $\mathrm{IV}_{R}$ in Figure $9(\mathrm{f})$, the vehicle travels close to the centerline of the road within the whole hairpin curve; in fact, this driving pattern is common on mountain roads.

\section{Risk Analysis of Road Crashes in Hairpin- Turn Sections}

The traffic volume on two-lane mountain roads is relatively low, which leads some aggressive and adventurous drivers to plan their path within the whole pavement width. When passing a turn in a curve-cutting pattern or to obtain a more adequate sight distance, an aggressive driver will occupy the opposite lane. In this scenario, vehicle track will intertwine with that of a vehicle in the opposite lane, causing a kinematic interference, that is, an area highly prone to road crashes, as shown in Figures 10(a) and 10(b).

By superimposing the distribution areas of the tracks in the two directions, the interference area between the leftturning tracks and right-turning tracks and its distribution along the hairpin-turn road section can be determined, as seen in Figures 10(c) and 10(d). For turn 2, the track interference occurs throughout half of the turn (i.e., left half of the turn), where the left-turning tracks that leave the turn significantly intertwine with the right-turning tracks that enter the turn. For turn 8, intertwining primarily occurs in the straight segments. An analysis of the track interference at the 11 hairpin curves indicates that track interference mostly and easily occurs at a turn between a left-turning vehicle that leaves the turn and a right-turning vehicle that enters the turn; that is, more significant track intertwining occurs in the left half of a turn.

A vehicle occupying the opposite lane will involve a heading-on collision with a vehicle in the opposite lane, as shown in Figure 11. In this scenario, a road crash will be more severe. During the field driving tests, the vehiclemounted Mobileye ${ }^{\mathrm{TM}} 630$ system recorded the lateral distances between the wheels and lane markings for both sides. Whether the vehicle occupied the opposite lane can be 


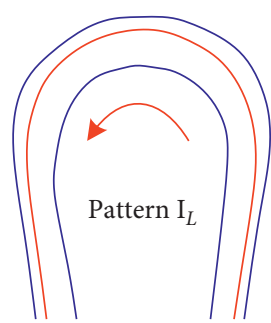

(a)

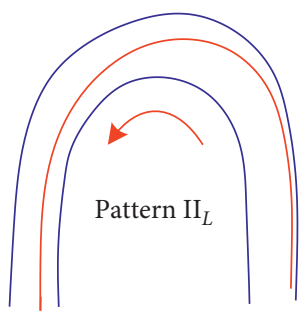

(b)

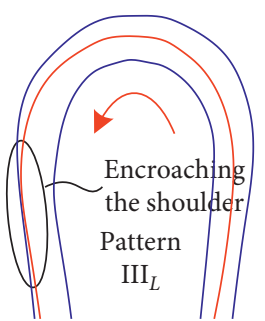

(c)

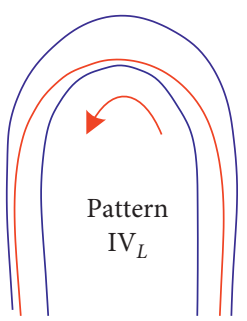

(d)

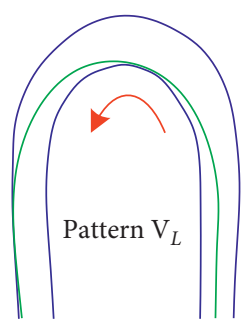

(e)

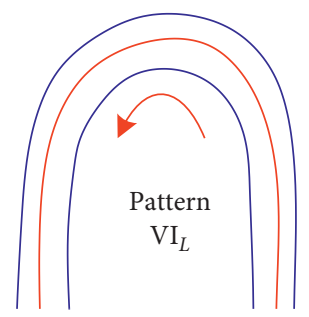

(f)

FiguRE 8: Track patterns at left-hand hairpin curves. (a) Pattern $\mathrm{I}_{L}$ : traveling in the legal lane; (b) pattern $\mathrm{II}_{L}$ : cutting a curve at the exit; (c) pattern $\mathrm{III}_{L}$ : occupying the opposite lane at curve entrance and occupying the shoulder when leaving the turn; (d) pattern $\mathrm{IV}_{L}$ : cutting a curve at the middle; (e) pattern $\mathrm{V}_{L}$ : cutting curve at the entrance; (f) following the road centerline.

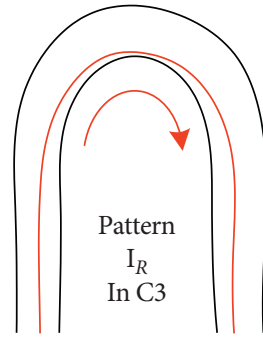

(a)

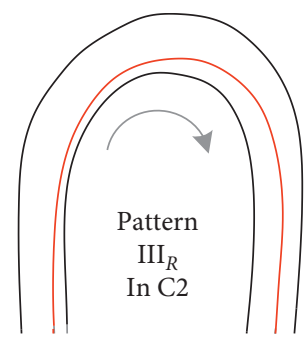

(d)

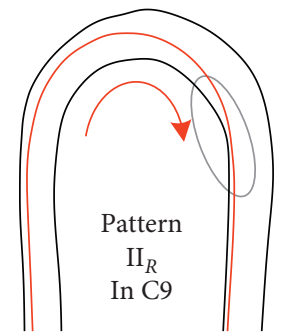

(b)

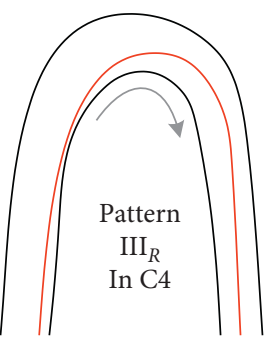

(e)

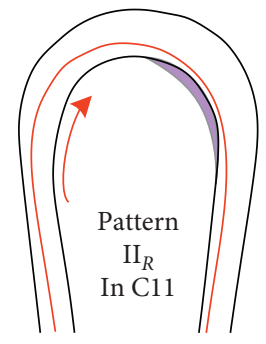

(c)

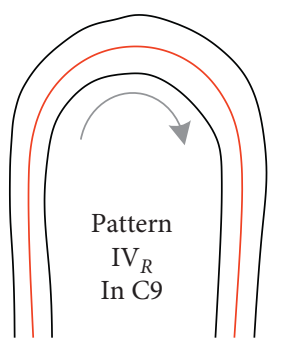

(f)

FIGURE 9: Right-turning track patterns at the hairpin curves. (a) Pattern $\mathrm{I}_{R}$ : curve-cutting in curve middle; (b) and (c) pattern $\mathrm{II}_{R}$ : cutting the curve when departing; (d) and (e) pattern $\mathrm{III}_{R}$ : cutting the curve at the entrance; (f) pattern $\mathrm{IV}_{R}$ : traveling in the middle of the road.
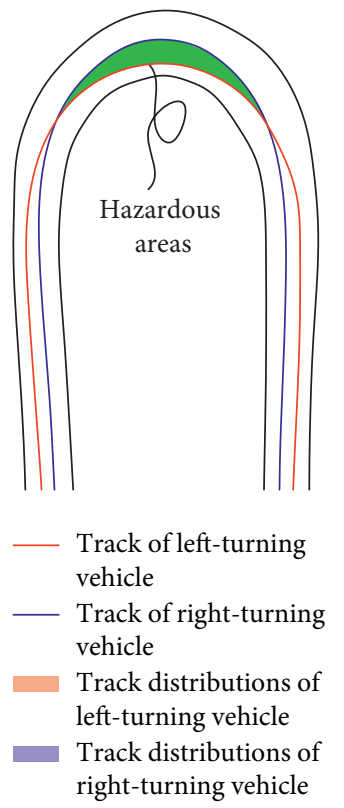

(a)

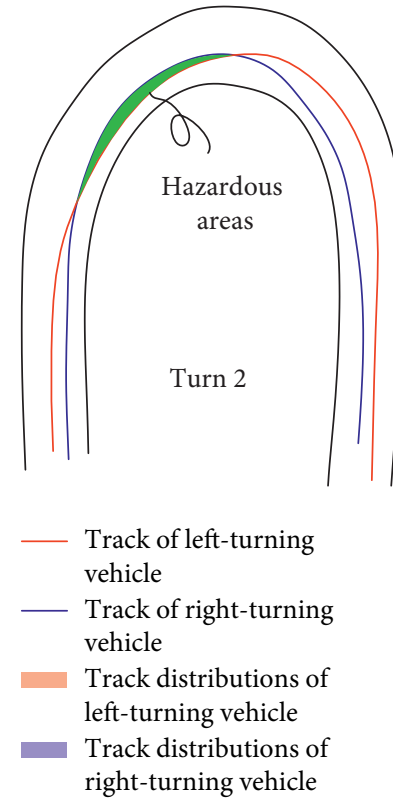

(b)

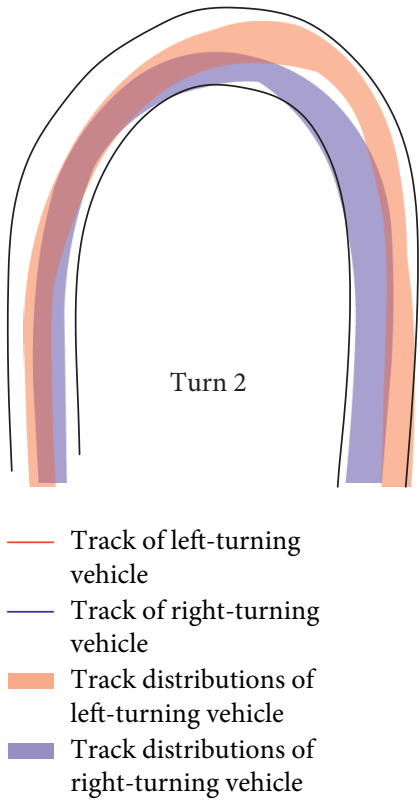

(c)

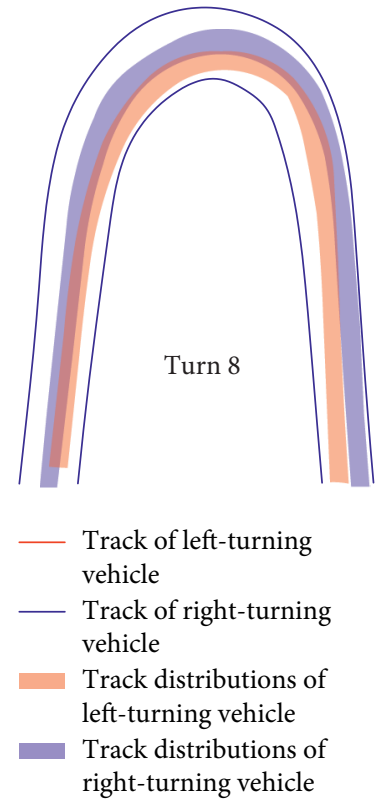

(d)

FigURE 10: The interweave behavior of vehicle track in different directions. (a, b) Intertwining and interference between left-turning tracks and right-turning tracks and $(c, d)$ intertwining between vehicle tracks in opposite lanes in hairpin-turn road sections. 


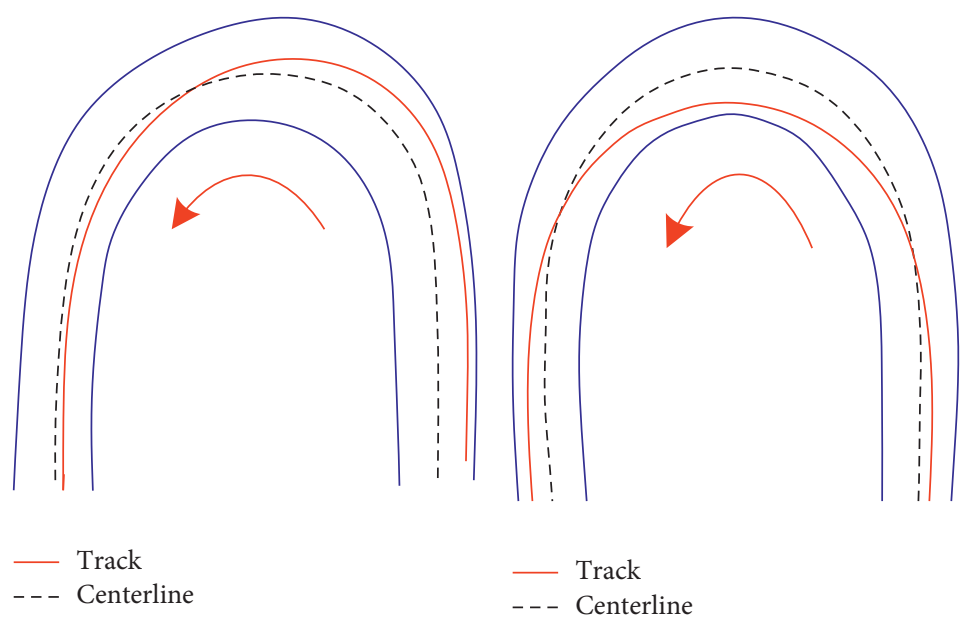

(a)

(b)

Figure 11: Vehicle occupying the opposite lane on hairpin curves.

determined based on the net lateral distance between its leftside wheels and the centerline of the road. Figure 12 shows the statistics of lane-occupying behaviors at the hairpin curves.

Figures 12(a) and 12(b) show the proportion of the drivers that occupied the opposite lane when entering each hairpin turn. Of all the left-hand turns, the highest proportion (greater than 90\%) of the drivers that occupied the opposite lane occurred at turn 2, and the lowest proportion (50\%) occurred at turn 11 . Mean proportion of the drivers that occupied the opposite lane at all the turns was $71.07 \%$. This finding suggests that occupying the opposite lane was a predominant behavior among the drivers when turning left into a hairpin turn. The proportion of the drivers who occupied the opposite lane when turning right into a hairpin turn ranged from $82 \%$ to $41 \%$, with an arithmetic mean of $59.5 \%$, which means that more than half of the drivers occupied the opposite lane. A comparison indicates that the proportion of the drivers that occupied the opposite lane was approximately $10 \%$ lower when negotiating a right-hand hairpin turn than left-hand hairpin turn; therefore, left-hand hairpin turns are associated with a relatively higher risk of road crashes.

Figure 12(c) shows the statistics of five kinds of laneusing behaviors, that is, occupying the opposite lane at curve exit $\left(\mathbf{O}_{\mathbf{A 4}}\right)$, traveling in the legal lane throughout the course $\left(\mathrm{LK}_{\mathrm{A1} 1-5}\right)$, occupying the opposite lane in the curve middle and curve exit $\left(\mathbf{O}_{\mathbf{A 3}-4}\right)$, occupying the opposite lane at curve entrance $\left(\mathbf{O}_{\mathrm{A} 2}\right)$, and occupying the opposite lane at curve entrance and curve middle $\left(\mathbf{O}_{\mathbf{A 2 - 3}}\right)$, which were observed in the drivers when turning left into hairpin turn. As demonstrated in Figure 12(c), the vehicle traveled in the legal lane less than $30 \%$ of the times, whereas the vehicle occupied the opposite lane at various locations of a hairpin turn in more than $70 \%$ of the time. The proportion of occupying the opposite lane was the highest during the exit from the turn, followed by that in the middle of the turn.

Figure 12(d) shows the lane-using behaviors observed on the right-hand hairpin turns, in which four lane-using behaviors are noted. The proportion of the drivers who drove in the legal lane throughout the course was the highest (40.5\%) because when making a right turn, the vehicle was on the inner side of the turn and its traveling path in the traffic lane was short. Thus, 59.5 percent of drivers occupy the opposite lane when they negotiate a hairpin curve, and this proportion is $10 \%$ lower than that on left-hand hairpin turns. Of the opposite lane-occupying behaviors, the proportion of occupying the opposite lane during the entry into the turn was the highest (26.86\%), which differs significantly from when the drivers made a left turn. This is because some drivers considered the sight distance within the lane to be inadequate due to obstruction by the mountain, and some drivers occupy the opposite lane to ensure an acceptable visual range.

Figure 13 shows the images captured by the front camera when the drivers were leaving downslope and upslope hairpin curves. As demonstrated in the figure, the vehicle was partially or even completely exposed to the opposite lane.

\section{Track Behavior Compared with General Curves}

The main purpose for drivers cutting a curve is to flatter the track radius within curve areas. The concept of equivalent radius $\left(R_{t e}\right)$ is proposed in this paper and can be calculated as follows:

$$
R_{t e}=\frac{1}{C_{t e}}=\frac{1}{(1 / n) \sum_{1}^{n} C_{t, i}}
$$

where $C_{t e}$ is the equivalent curvature of track, which means the average of track curvature within the range of circular curve, $C_{t, i}$ is the track curvature at point $i$, and $n$ is the number of track coordinate points falling within the scope of circular curve, that is, the data points of track within SC-CS range in Figure 14(a). If the equivalent radius $R_{t e}$ is greater than the design radius of a curve, it indicates that the driver 

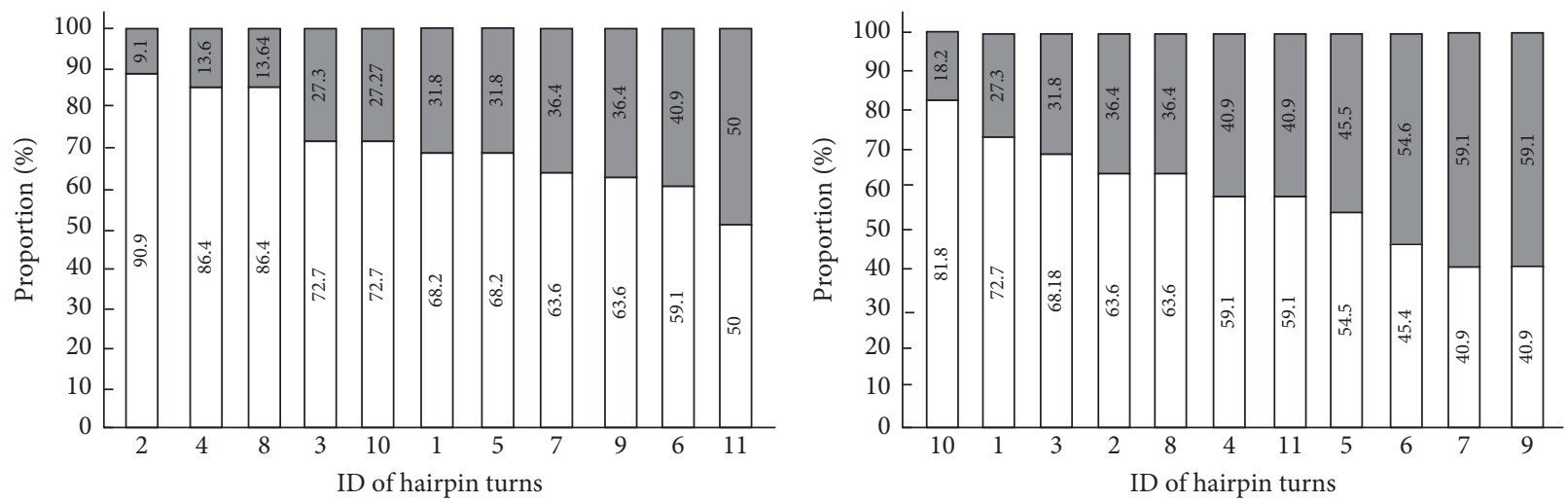

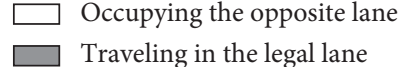

(a)

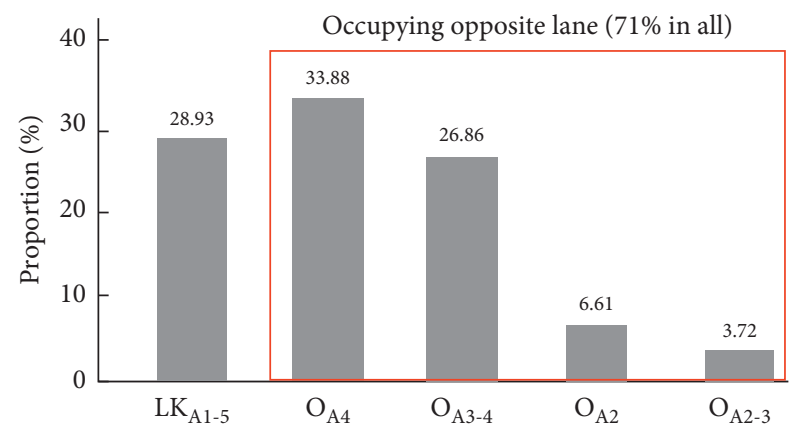

(c)

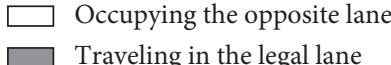

(b)

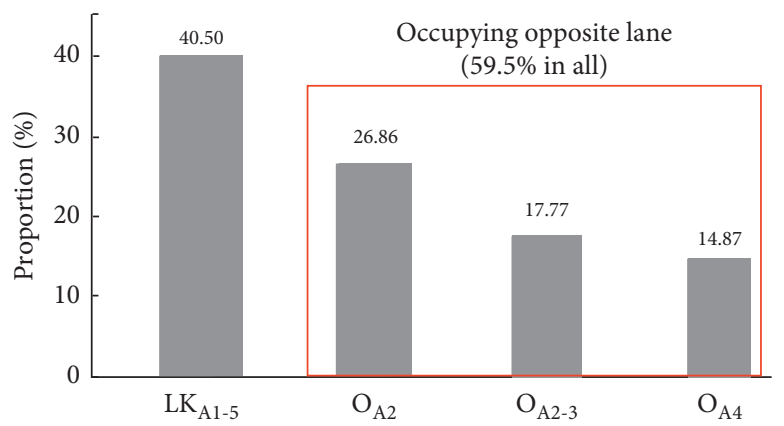

(d)

FiguRE 12: Opposite lane-occupying behaviors and their proportions. (a) Left-hand turns. (b) Right-hand turns. (c) Left-hand turns. (d) Right-hand turns.

can acquire a benefit of flattering the track by cutting the curve, and the average track radius within the curved range can be increased.

For each hairpin turn, calculate the value of $C_{t e, j}, j=1$, ..., $m$, where $m$ is the number of effective tracks, and then the maximum and minimum of equivalent radii, $R_{t e m a x}$ and $R_{\text {temin }}$, are extracted, as shown in Figure 14(b), respectively, to represent the most radical and conservative track choice behavior, and then calculate the radius increment $\triangle R_{t}$ :

$$
\Delta R_{t}=R_{\text {temax }}-R_{d}
$$

Hairpin curves have the significant characteristics of minimum radius, large deflection angle, and steep slope. In order to confirm whether the track behavior on hairpin curve is significantly different from that on general curved section, we compare and analyze the track data of three general curve sections. The horizontal alignment parameters of the three curves are curve 12: radius $35 \mathrm{~m}$, deflection angle $64.4^{\circ}$; curve 13 : radius $80 \mathrm{~m}$, deflection angle $37.12^{\circ}$; curve 14 : radius $120 \mathrm{~m}$, deflection angle $29.2^{\circ}$. Because the three curves have different radius, it is difficult to accurately describe the cutting effect only by using the increment in track radius $\triangle R_{t}$, so the dimensionless parameter is added:

$$
P \Delta R_{t}=\frac{\Delta R_{t}}{R_{d}} .
$$

Namely, the ratio between the increment in track radius and the design radius of curve.

Figure 15 shows the track measured on these three curves and marks the centerline of the road. It can be observed that the distribution area of the track is basically the same as the lane width, which indicates that the path selection behavior has strong discreteness; in contrast, the dispersion of left turns track is higher than that of right turns. The track of some right-turning vehicles is close to the road centerline, and if the vehicle body width is considered, the vehicle has occupied the opposite lane. Meanwhile, there are a large number of left-turning vehicles in the opposite lane, especially in the middle of the curve.

Figure 16 shows the $P_{\triangle R t}$ histogram of three types of hairpin curves (deflection angle $(\mathrm{DA})>180^{\circ}, \mathrm{DA} \approx 180^{\circ}$, and $\mathrm{DA}<180^{\circ}$ ) and three general curves. It can be seen from the figure that $P_{\triangle R t}$ of hairpin curves is significantly lower than that of three general curves whether it is left turn or right turn, which indicates that the track shape on hairpin curves presents obvious cutting behavior characteristics, encroaching the opposite lane and hard shoulder. But as far 


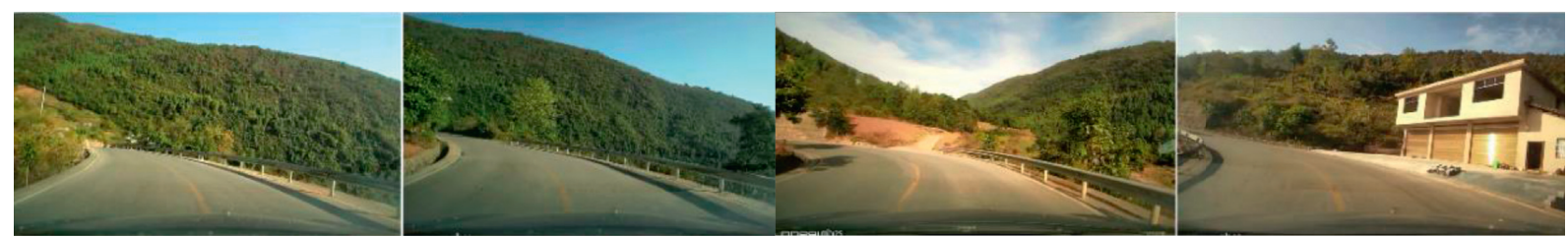

(a)

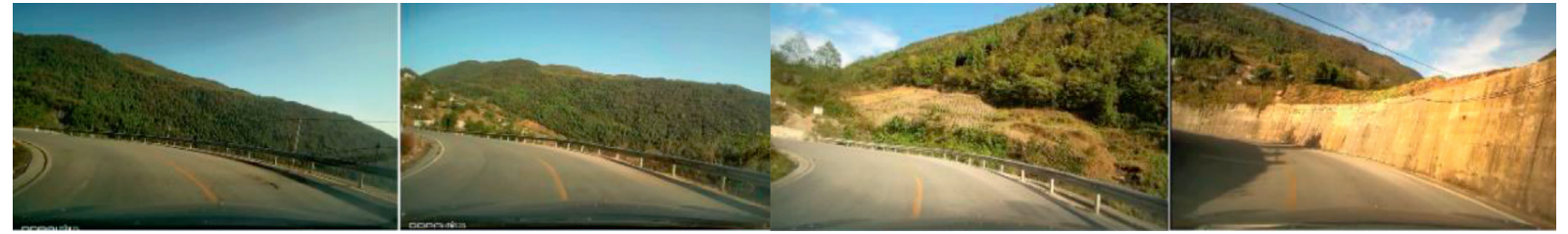

(b)

Figure 13: Exposure of the vehicle to the opposite lane. (a) Driving on downslope hairpin curves. (b) Driving on upslope hairpin curves.

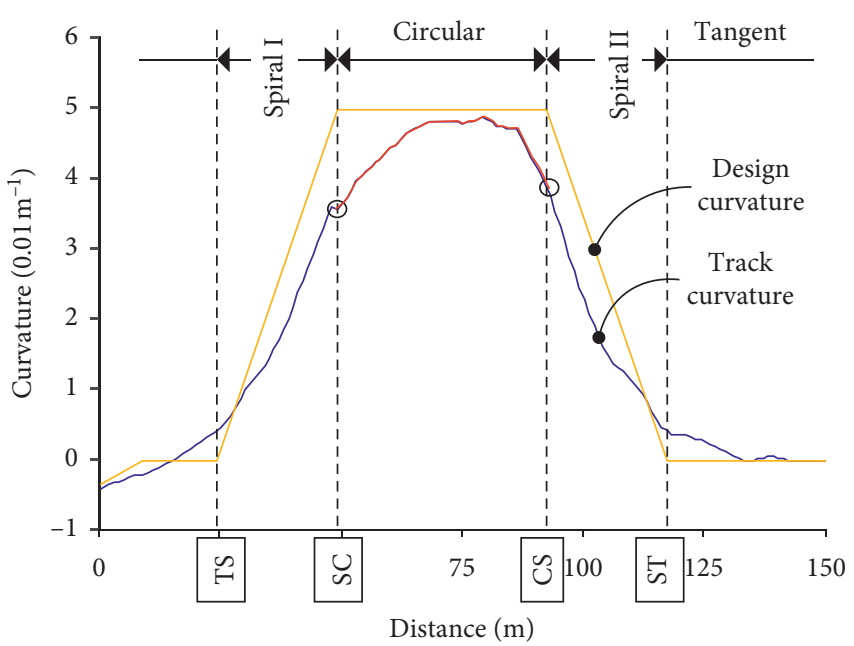

(a)

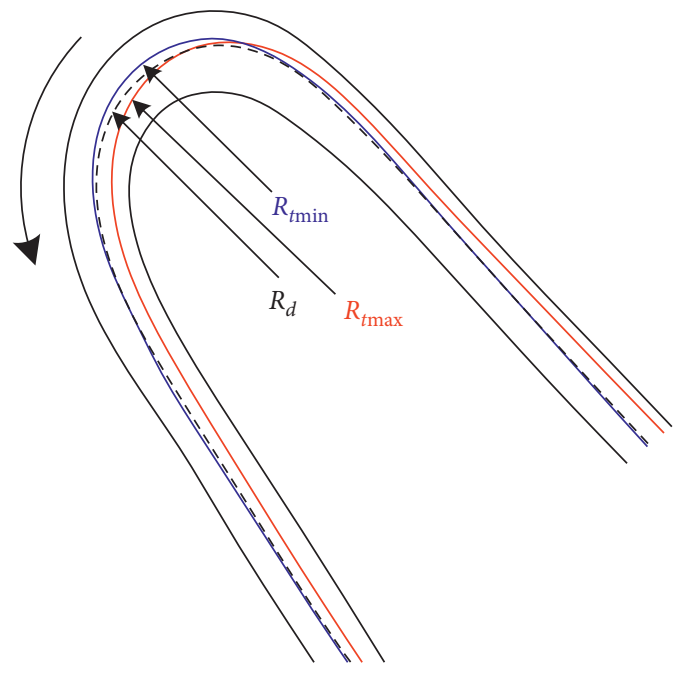

- Edge line - . - Centerline

- Track

(b)

FIGURE 14: Calculation and illustration of track equivalent radius. (a) Calculation of track equivalent curvature and (b) illustration of track equivalent radius. 


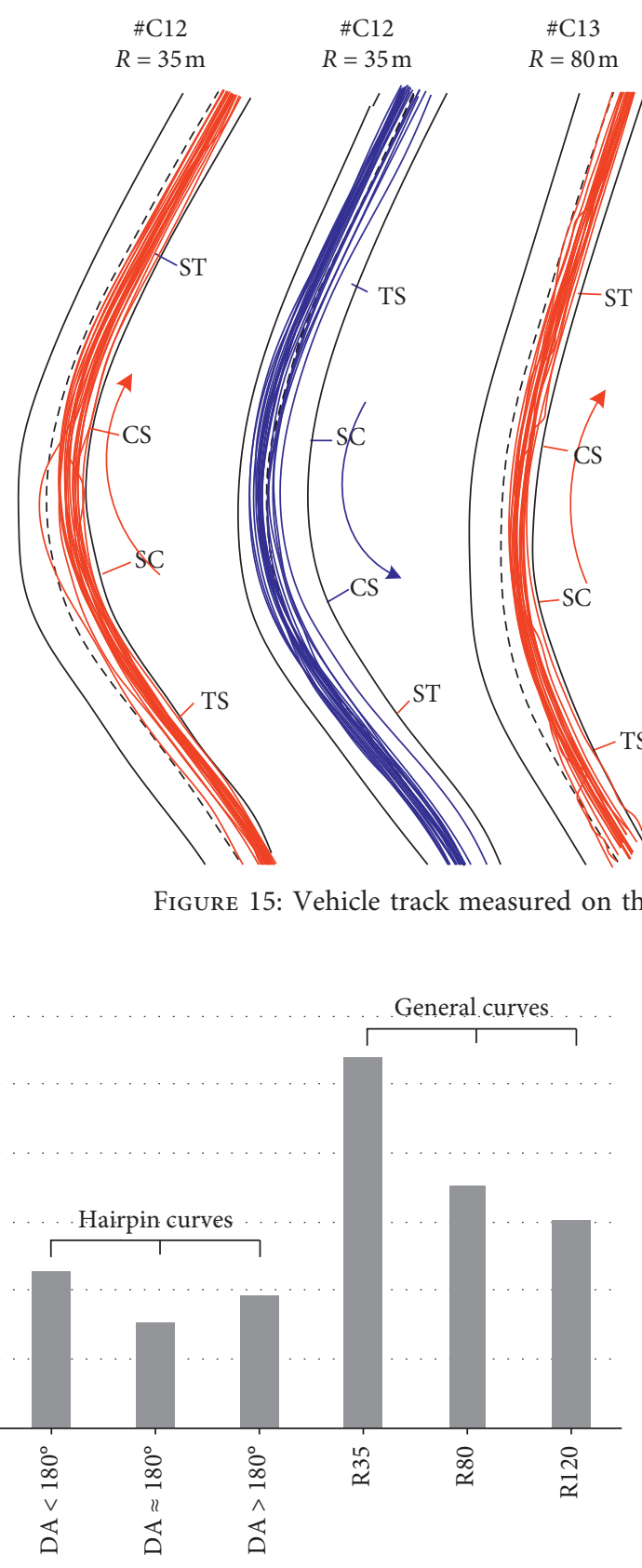

(a)

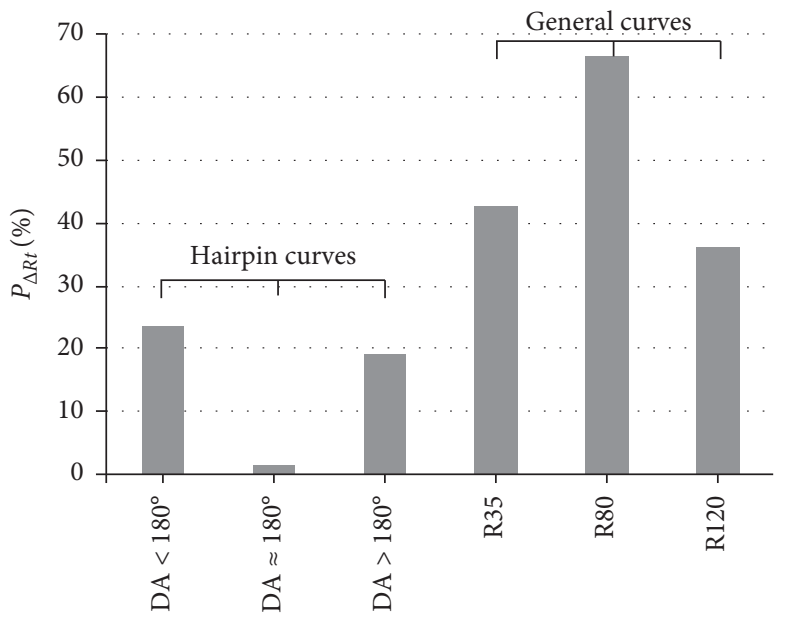

(b)

Figure 16: Histogram of $P \triangle R t$ for hairpin curves and general curves. (a) Left-hand curves; (b) right-hand curves.

as the effect of cutting effect is concerned, it is not as good as the general curves with low deflection angle.

\section{Conclusions}

Hairpin curve is an alignment combination that is commonly employed in geometry design of mountain roads, particularly ridge-crossing route for two-lane mountain roads, and they are an important feature that can help identify dangerous driving conditions on mountain roads. However, driving behaviors at hairpin curves remain ambiguous. Field driving tests were conducted and track data were collected at 11 hairpin curves and three general curves.
Track morphology and patterns were analyzed, as well as the road crash risks and mechanisms. The main findings are summarized as follows:

(1) Tracks at the hairpin curves, regardless of left-hand or right-hand curves, were relatively highly discrete and clustered, which indicates a difference in the track-selecting behaviors among drivers.

(2) Curve cutting was a typical track pattern observed at hairpin curves and was also an important factor that induced a vehicle to occupy the opposite lane. Curve cutting was observed at both left and right turns. Based on the location of the cutting point, curve- 
cutting behaviors can be classified into three types, namely, cutting at curve entry, cutting at curve middle, and cutting at curve exit. The latter two curve-cutting patterns are also the most common behavior on racing tracks. When cutting at curve exit, a driver will first control the vehicle close to the outer side at the bend entry.

(3) Based on the lateral positional relationships between tracks and lane markings, tracks at the hairpin curves are classified, and six patterns are identified for left-hand curves and four patterns for right-hand curves. Of all track patterns, a vehicle moves within its legal lane throughout the course in only one pattern, and in the remaining patterns, the vehicle occupies the opposite lane or the right-side shoulder.

(4) When passing a right turn by curve cutting, the drivers may occupy the right shoulder at curve entrance, curve middle, and curve exit and lead to collision with the mountain or roadside guardrail. When turning left into a hairpin turn, some drivers occupied the right shoulder on curve exit, resulting in the vehicle running off the road or colliding with the guardrail.

(5) Vehicles partially or completely occupied the opposite lane at a hairpin turn due to behaviors of curve cutting and driving in the middle of the road. The tracks in the two driving directions intertwined with one another in curve area and tangent, which may cause a risk of head-to-head collision.

(6) More than $70 \%$ of the drivers occupied the opposite lane when turning left into hairpin curves, and for right turns, the proportion has dropped by about 10 percent; namely, $59.5 \%$ of drivers occupy the opposite lane. For left-hand curves, drivers frequently occupied the opposite lane at curve exit, while for right-hand curves, the location where driver frequently occupied opposite lane is the curve entrance.

The limitations of this study are as follows: the risk analysis of our paper does not offer an explicit link between lane position and crash frequency, because in China, the traffic accident data of each road is not open to the public, so we cannot get the traffic accidents on the test road in recent years. If we can obtain the crash data at each study site, we can investigate the contributing factors to the crash and may help to more explicitly link lane position to safety performance.

\section{Data Availability}

The data used to support the findings of this study are available from the corresponding author upon request.

\section{Conflicts of Interest}

The authors declare that they have no conflicts of interest.

\section{Acknowledgments}

This research was supported by the National Key R\&D Program of China (2018YFB1600500).

\section{References}

[1] J. Xu, X. Luo, and Y. M. Shao, "Vehicle trajectory at curved sections of two-lane mountain roads: a field study under natural driving conditions," European Transport Research Review, vol. 10, no. 1, pp. 1-16, 2018.

[2] D. Paolo, "Experimental analysis on hairpin curves," The Baltic Journal of Road and Bridge Engineering, vol. 5, no. 3, pp. 148-155, 2010.

[3] R. Chapuis, A. Potelle, and J. L. Brame, "Real-time vehicle trajectory supervision on the highway," The International Journal of Robotics Research, vol. 14, no. 6, pp. 531-542, 1995.

[4] C. Llorca, A. T. Moreno, A. García et al., "Daytime and nighttime passing maneuvers on a two-lane rural road in Spain," Transportation Research Record: Journal of the Transportation Research Board, vol. 2358, pp. 3-11, 2013.

[5] V. Kanagaraj, G. Asaithambi, T. Toledo et al., "Trajectory data and flow characteristics of mixed traffic," Transportation Research Record: Journal of the Transportation Research Board, vol. 2491, pp. 1-11, 2015.

[6] N. Raju, P. Kumar, A. Jain et al., "Application of trajectory data for investigating vehicle behavior in mixed traffic environment," Transportation Research Record, vol. 2672, no. 43, pp. 122-133, 2018.

[7] T. M. Deng, X. Luo, Y. M. Shao et al., "Track patterns and curve cutting behavior on curved sections of mountain roads," Journal of Southeast University(Natural Science Edition), vol. 49, no. 3, pp. 388-396, 2019.

[8] Q. Y. Peng, J. Xu, Q. Luo et al., "Effect of horizontal curves design on track and speed of passenger car," Journal of Tongji University (Natural Science), vol. 40, no. 1, pp. 45-50, 2012.

[9] J. Xu, Q. Luo, Q. Y. Peng et al., "Influence of spiral setting on vehicle speed on curve," China Journal of Highway and Transport, vol. 24, no. 1, pp. 25-33, 2011.

[10] H. B. Shu, M. Shao Yi, and J. Xu, "Effects of geometric features of highway horizontal alignment on steering behavior of passenger car," Journal of Vibro Engineering, vol. 18, no. 6, pp. 4086-4104, 2016.

[11] P. Spacek, "Track behavior in curve areas: attempt at typology," Journal of Transportation Engineering, vol. 131, no. 9, pp. 669-676, 2005.

[12] E. J. Fitzsimmons, S. S. Nambisan, R. R. Souleyrette, and V. Kvam, "Analyses of vehicle trajectories and speed profiles along horizontal curves," Journal of Transportation Safety \& Security, vol. 5, no. 3, pp. 187-207, 2013.

[13] P. Spacek, "Track behaviour and accident occurrence in curves on two-lane highway in rural areas," in Proceedings of the 2nd International Symposium on Highway Geometric Design, pp. 288-298, Transportation Research Board, Mainz, Germany, 2000.

[14] S. R. Geedipally and M. P. Pratt, "Predicting the distribution of vehicle travel paths along horizontal curves," Journal of Transportation Engineering, Part A: Systems, vol. 143, no. 7, Article ID 04017021, 2017.

[15] F. Mauriello, A. Montella, M. Pernetti, and F. Galante, “An exploratory analysis of curve trajectories on two-lane rural highways," Sustainability, vol. 10, no. 11, p. 4248, 2018. 
[16] A. Boruah and A. K. Maurya, "Vehicle's lateral placement on horizontal curves: a literature review," Transportation Research Procedia, vol. 48, pp. 1325-1333, 2020.

[17] D. Trump and J. Parkin, "Equestrian road collisions: an analysis of the collision record, riding behaviours and experiences," Safety Science, vol. 123, Article ID 104523, 2020.

[18] B. Wali, A. J. Khattak, and J. J. Xu, "Contributory fault and level of personal injury to drivers involved in head-on collisions: application of copula-based bivariate ordinal models," Accident Analysis and Prevention, vol. 110, pp. 101-114, 2018. 